\title{
Voltage control of bidirectional DC-DC converter with constant power source
}

\author{
Chimdi Tadesse Girma, song chi \\ State key laboratory and intelligence of electrical equipment Hebei university of technology Tianjin china
}

\begin{abstract}
Bidirectional DC/DC converters are used in the interface of the battery bank and the high voltage direct current terminal of an inverter. The performance of the system depends on the control of voltage and current across the circuit. Voltage control in eliminates the need for changing the control loop when the power supply is changed to the alternating current source. The report explains the constant power supply voltage control. The diagram for the bidirectional $\mathrm{dc} / \mathrm{dc}$ converters is analysed and the mathematical representations are given. The dynamic performance of the circuit is calculated to give the efficiency of the system in DClink voltage control.
\end{abstract}

\section{INTRODUCTION}

The distribution of the current in the three axes needs measurement to ensure correct stabilization and conversion. The electrical measurements taken have determined the con- version process and the rectification process. The factor of safety determines the amount of the factor of amplification. The microchip has the programs to determine the level of amplification and the conversion process [1].

An electric field is made up of lines that are perpendicular to the points with equal electric potential on the surface. If an electrically charged body moves over the surface, no electrical work is required. Due to the equipotential surfaces have a uniform distribution of the electric potential and its perpendicular to the direction of the electrical field lines at any point the electric field lines originate from the positive side of the electric charge and end at the negative.

The electric charges produce vector fields that make up the electric field. The source of the electric field is single or multiple charges. The lines of force aid in the visualization of the electric field. Work must be done to move charges across the points with different electric potential. The electric potential is described as the work done per unit charge. The lines of the equipotential force fields show the force field. The electric fields are mapped if the equipotential lines are drawn. The experiment aimed to trace the equipotential surfaces thus draw the electric field lines produced by different potential differences.

During the test, a sheet of paper was immersed in the plastic box filled with water. Two metal objects were placed on the paper, and their positions were traced. The metal objects were connected to the power, and the DMM was set to $2 \mathrm{~V}$ range. The electricity supply was turned on and the DMM adjusted to $10 \mathrm{~V}$. A hollow cylinder conductor was placed on the adjustable ring, and the probe was set vertically in the water at any point and the DMM reading recorded. The probe was moved, and another point with the same DMM reading marked. That was done to mark several points. The paper was removed from

the plastic box to dry. The above was done with two hollow cylindrical conductors $10 \mathrm{~cm}$ apart. Rectangular wires were used to mark the equipotential points. The paper dried and the points were joined with a dotted line. Use of the points constructed the field lines.

The equipotential points lie at the points with the same electric potential [2]. The dash lines form curved lines about the source of the charge. The electric field lines are drawn a perpendicular to the equipotential lines. However, during the experiment the following sources of error occurred:

Slight operation of the conductor resulted in wrong points marked Error in setting the probe

The Reading from the DMM could be varying due to the drifting of the characteristics of the equipment

Error in the tracing of the equipotential points the electric field lines were drawn the perpendicular to the equipotential points. The dashed line that traced the equipotential lines formed a circular line. No work is done to move charges across the points with the same electric potential. The electric potential is strongest at the points near the charge and it weakest at the points far from the source of the charge.

The points with the same potential formed a circle about the source of the charge. This shows that the electric potentials at those points were equal. No work was required to move the charge across that points. The location of the points would change if the different conductor were used. This is because; different materials have different resistance and inductance.

This problem occurs in most weigh power measuring devices. In most weigh bridges they measure the vertical load distribution forgetting the axle load of the current 
source. If the axle load distribution is high, then it means that the rear wheels are overloaded, and this can result in damage to the roads.

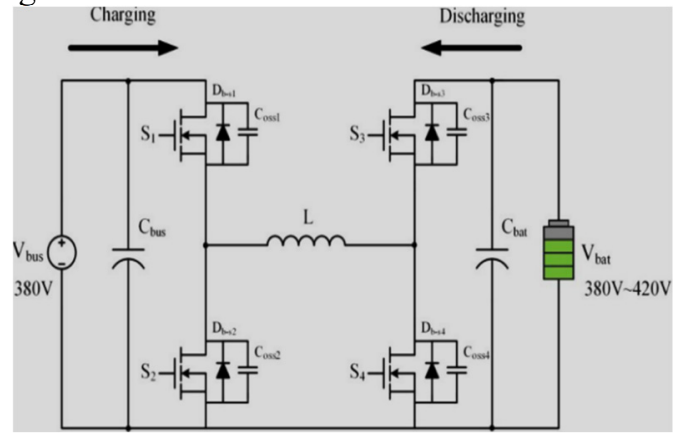

\section{EXPLANATION}

The microchip has two different voltage inputs. The intent is leaving a single entry that allows control of motors, facilitating and tracking the path on the $\mathrm{X}$-axis and $\mathrm{Y}$. the system has to behave as a system of first order. This is why it is intended that the input signals to the system is only one (Single Input- Single Output, SISO) and not different input signals at once (Multiple Input - Multiple Output, MIMO). Considering that if the voltage applied to each of the motors is the same, the angle is zero, and if instead each of the applied voltages the engine is different, the output will be a variation in angle.

The program communicates with a computer through electric cables, waterproof hoses.

The ordinate executes a program in the Processing language. This program reads the instructions of a Lynx motion AL5D PLTW controller and uses them to calculate the values. The program also has a graphical interface with several sliders that control the servo motors in the ROV, the bidirectional dc- dc converter ic clamp and the other diverse accessories that incorporate the controllers. The outdoor control station also has a monitor to view the image that are broadcast on the three cameras on board the controller.

The power amplifier provides the necessary current to the motors and in this case, is used an IC L293D, which is an $\mathrm{H}$ bridge that allows the change of direction of the motors connected to the fans for controlling the rotation of the bidirectional dc-dc converter [3].

They are non-linear systems where several parameters of the model are little or no known (under the damping matrix, the masses added ...) and may vary during operation. Also, some effects cannot be properly integrated model (effect and umbilical for semiautonomous control, effects of vortex flows). Finally, the vehicle is subject to frequent disturbances such as waves and shocks. These unfamiliar terms and these disturbances affect and varied on the vehicle with their ap- appearances. On small marine (mini ROV mini AUV) control approaches used on high inertia, vehicles do not feel sufficient to ensure acceptable performance 1 . The external disturbances (exogenous system), which are usually due to the external environment such as an increase in temperature affect the viscosity of water, various water stream. 2. Internal disturbances, which are usually due to modelling errors andmeasurements from the sensors. In our case, the ROV moves with low speeds. Is $\sim \mathrm{g}$ an internal term uncertainty or externally due to modelling errors or environmental disturbances. In general,we do not know $\sim \mathrm{g}$ explicitly, but some information on $\sim \mathrm{g}$ will be required. We consider the given system, linked to the dynamics of ROV.

The study of the voltage control usually leads to a study of disturbed systems. The analysis consists of analysing the origin of the stability of the nominal system. To ensure this voltage control property, we show that the knowledge of the explicit form of the Lyapunov function is not necessary. It is assumed that the origin is an equilibrium point for the unperturbed system (fig 2).

There is a need for this sensor to identify the disturbances during bidirectional $\mathrm{dc}-\mathrm{dc}$ converter operation [4]. The bidi- reactional dc-dc converter needs to have a recognition of the contour of the pool or pond, to detect the place in which you must download the material collected. For these three infrared distance sensors (1 and 2 GP2D12 GP2D120), located on the front and sides of the bidirectional dc-dc converter are implemented. (fig3)

There is a need for this sensor to identify the disturbances during bidirectional $\mathrm{dc}-\mathrm{dc}$ converter operation. The bi-directional dc-dc converter needs to have a recognition of the contour of the pool or pond, to detect the place in which you must download the material collected.

In the bidirectional dc-dc converter two fans connected directly to DC motors V 3.5 W 9 are installed, for a speed of $0.42 \mathrm{~m} / \mathrm{s}$, while taking control in the direction of operation of the bidirectional dc-dc converter, so that when both motors are activated simultaneously in the same direction, the bidirectional dc-dc converter advances straight and if the turning direction is inverted in one of them, can be rotated to the right or left as appropriate. The fans are located on the back of the structure.

The maximum rate of discharge $=$

The number of batteries in parallel $x B C$

(The number of modules in parallel $x$ Imp

Four $x$ 180Ah/

$=17.14$ hours

$$
4 \times 3 A
$$

The rate of discharge calculated as follow

$$
\text { The daily load (Ah) }
$$

The number of batteries in parrallel $x B C(A h$

$$
\begin{gathered}
\text { Therefore, the DOD }=\frac{204.16 \mathrm{Ah}}{4 \times 180 \mathrm{Ah}} \times 100 \\
=28 \% .
\end{gathered}
$$

The battery cells have a maximum output when slightly tilted to face the side with direct sunlight. A tilt angle of 


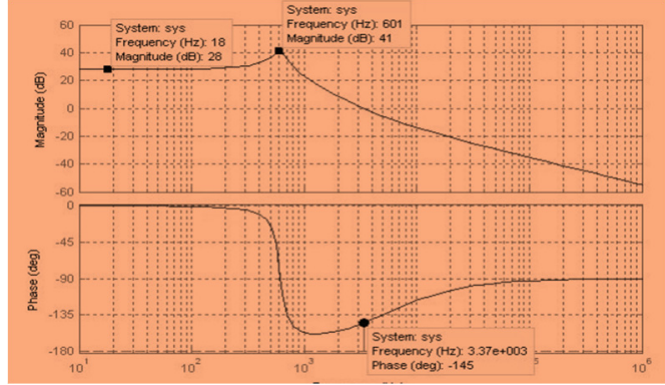

Figure 2: Open loop buck converter response

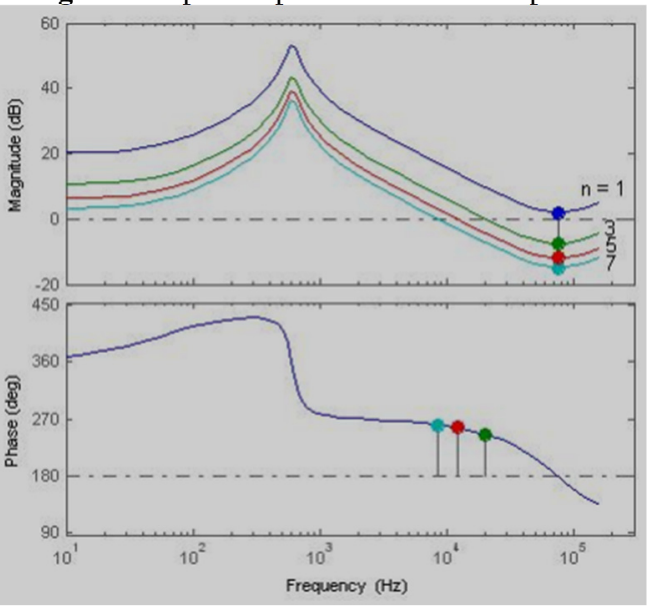

Figure 3: current loop bode plot - current mode control [W X Y Z] = linmod('buck_fullsmallsignal'); sys $=\mathrm{ss}(\mathrm{W}, \mathrm{X}, \mathrm{Y}, \mathrm{Z})$;

bode(sys, $\{10,10 \mathrm{e} 9\}$ );

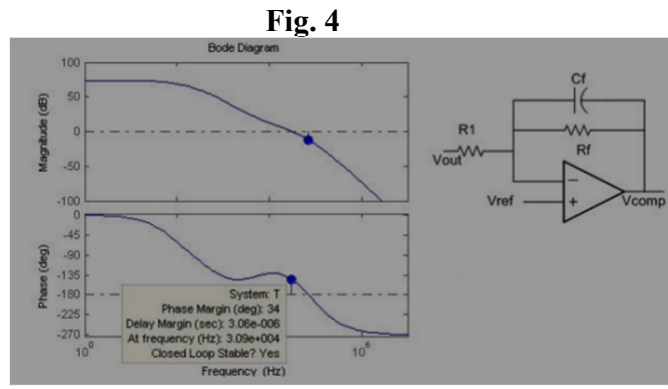

Figure 5: Compensated control to output loop and

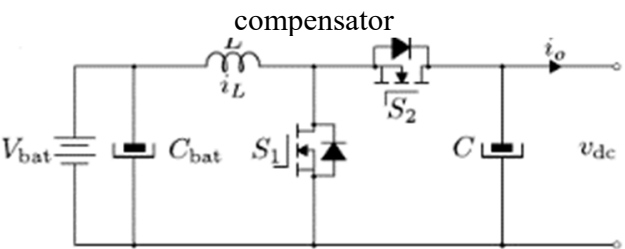

Fig. 6.

around 20 degrees is required for maximum output. A flat roof is the most suited for maximum output of the battery cells as the solar can be mounted on a frame and tilted at different angles depending on the position of the sun. The Bode graphs are generated with the following MATLAB code (fig4)

The area of the capacitor plates also affects the output of the battery cells systems. The standard recommendations are each 1000 watts of the battery cells module require a collector area of 100 square feet. This calculation is extended in the project to determine the size of the battery cells to be installed on the classroom rooftop.

\section{THE BATTERY CELLS ARRAY SIZING.}

The open circuit voltage required $\left(\mathrm{V}_{O C}\right)$ is $22.2 \mathrm{~V}$

The maximum daily load current is 204.16Ah The required short circuit $\left(\mathrm{I}_{S C}\right)$ current is $3.230 \mathrm{~A}$ The module output is $3 \mathrm{~A} \mathrm{X} 6 \mathrm{~h}=18 \mathrm{Ah}$

The total number of hours the classroom is under radiation at $1000 \mathrm{~W} / \mathrm{m}^{2}$ is 6 hours.

The selected module is the Sundance solar panel which is $60 \mathrm{~W}$ AND I $m p$ is $3 \mathrm{~A}$.

The calculations of the modules in both parallel and series

connection is as shown below

The number of modules in series is $=$

$$
\frac{\text { Nominal system volatge }(V)}{\text { Nominal module voltage }(V}
$$

Therefore, the total number of modules in series connection is $=\frac{24 v}{12 v}$

The number of modules in parallel modules is calculated as shown

$$
\frac{\text { the daily load demand }(A h)}{\text { module outpu(Ah)xTDFXDF }}
$$

Therefore, the number total number of modules in a parallel connection is

$$
\frac{204.16 \mathrm{AH}}{18 \mathrm{Ah} \times 0.9 \times 0.9}=14
$$

The array configuration of the modules in the project is 14 in parallel $x 2$ in series. For a deep cycle battery, the maximum charge rate is supposed to be at $\mathrm{C} / 5$ while the discharge depth is not supposed to drop below $80 \%$. This ensures that the battery maintains longa Per life.

The calculations for the depth of discharge and the maxi- mum rate of discharge is as shown below.

The maximum rate of discharge

$$
\begin{aligned}
& =\frac{\text { the number of batteries in parallelXBC }}{\text { THE NUMBER OF MODULES IN PARALLEL Ximp }} \\
& =\frac{\text { Four } x 180 \mathrm{AH}}{14 X 3 \mathrm{~A}} \\
& =17.14 \mathrm{hours} .
\end{aligned}
$$

The rate of discharge is calculated as follows $=$ The daily load $(A h)$

the number of batteries in parallel $x B C(A h)$

Therefore, the DOD $=\frac{204.16 \mathrm{Ah}}{4 \times 180 \mathrm{Ah}} \times 100$ $=28 \%$.

The figure (6\&7) shows the dc/dc converter circuit.

\section{VOLTAGE CONTROLLERS}

The design of the controller will allow the possibility of further modifications and additions, which will be mechanical structure. Control of the prototype will be made using a webcam which will allow recognition of the path followed by a particular object, and recognition of a predetermined path to follow within the environment. Determining the type of control that meets the requirements to follow a path through the device is part of the work and evaluation the proponents made in the development of this work.

The electrical control scheme of the inverted 
pendulum control should have the following functional units:

- Electric thrusters that provide the horizontal operation and vertical operation of the inverted pendulum.

- Control servo motors.

- A voltage converter to power the servo motors.

- A control programs.

- A computer control outside.

- A general switch with a 25 Amp fuse

Finally, a control system, which satisfied the conditions in- lamented displacement necessary to follow a predetermined object using Classical control.

\section{VOLTAGE DELTA}

The employee control for the proper functioning of our proto- type is the digital PID (proportional-integralderivative), which has been implemented in the microcontroller ATmega328. The PID controller responds to the following equation (fig 8)

Where e $(t)$ is the error signal $u(t)$ is the control input of the process. $\mathrm{Kp}$ is proportional gain; $\mathrm{Ti}$ is the integral time constant and $\mathrm{Td}$ is the derivative time constant. With this type of control can be seen that by varying each of the constant in the adaptive PID control, our system improves significantly, through the proportionality constant insured until you have achieved greater precision. When the direction approached the desired value, control comes into action comprehensive error which reduced the proportional control to position the vehicle at the precise point.

\section{MICROCONTROLLER PROGRAM FOR THE MACHINE}

The ordinate executes a program in the Processing language. This program reads the instructions of a Lynx motion AL5D PLTW controller. The power amplifier provides the necessary current to the motors and in this case, is used an IC L293D, which is an H bridge that allows the change of direction of the motors connected to the fans for controlling the rotation of the controller.

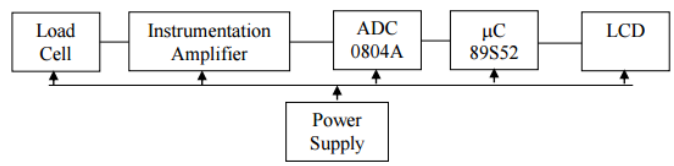

Fig 7

$$
u(t)=K_{p} e(t)+\frac{K_{p}}{T_{i}} \int_{0}^{t} e(t) \partial t+K_{p} T_{d} \frac{\partial e(t)}{\partial t}
$$

Fig 8

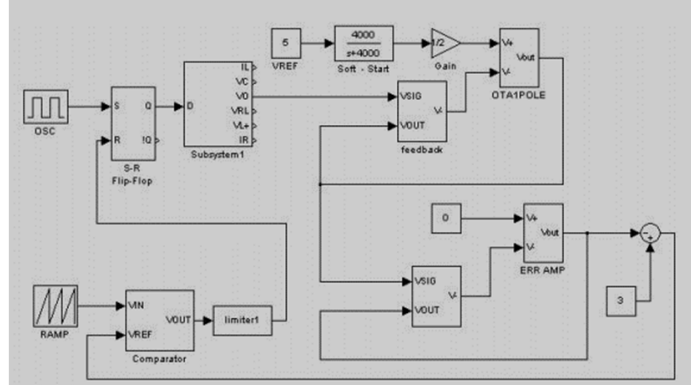

Figure 9: full Simulink model of voltage mode control

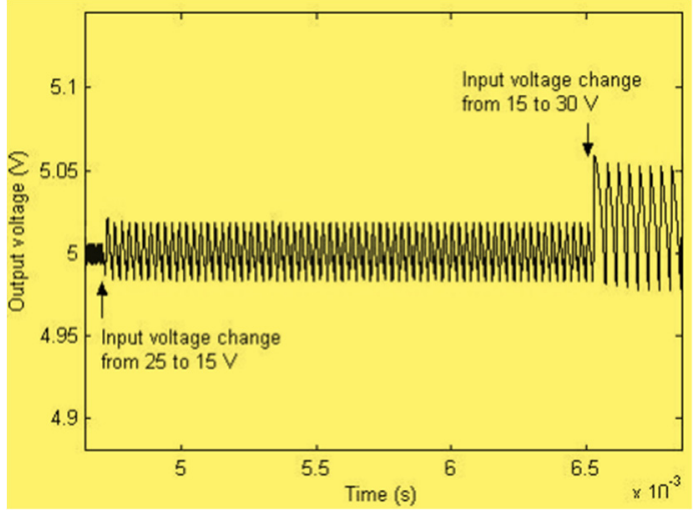

Figure 10: Voltage mode response to line variation

The flow chart below shows the general format and the procedure of the working principle of the machine

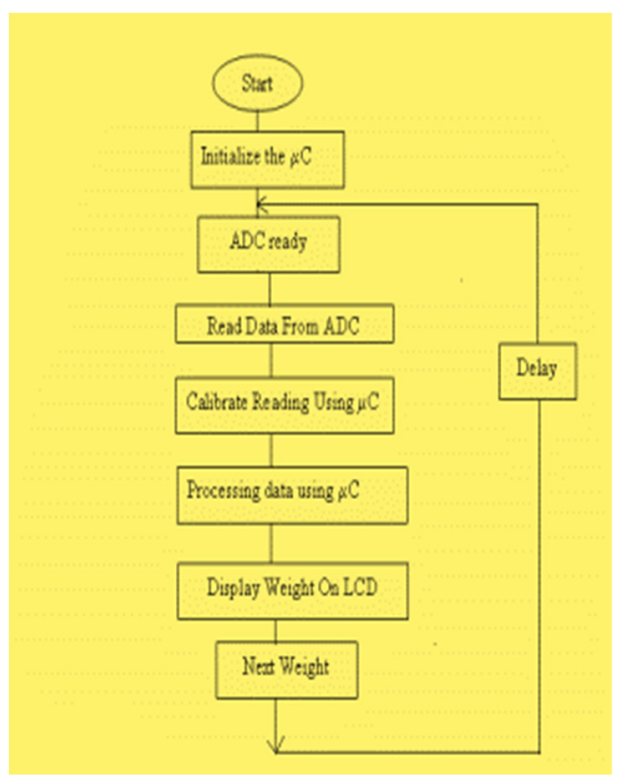

\section{CONCLUSION}

Voltage controllers should have correct transducers to measure the input and the output voltages and send feedback to the microchip for stabilization of the currents. The currents and voltages in the components are taken as vectors. The $\mathrm{X}$ and $\mathrm{Y}$ axes in the vector diagram give the magnitude and the direction respectively and the Z-axis gives the direction of the current in the vertical direction.the microchips have measuring mechanisms which have piezo-electric sensors. the transducers produce current when triggered by pressure the circuit divides the current at the input for measurement. 


\section{ACKNOWLEDGMENTS}

I would like to thank Mustafa AL rayah Hassan for his great support to finish my research successfully

\section{REFERENCES}

1. Chen, Dong, Lie $\mathrm{Xu}$, and Liang Zhong Yao. "DC voltage variation based autonomous control of DC microgrids." IEEE transactions on power delivery 28.2 (2013): 637-648.

2. Chen, Yu-Kai, et al. "Design and implementation of energy management system with with fuzzy control for DC microgrid systems." IEEE Transactions on Power Electronics 28.4 (20131563-1570.

3. Dong, Dong, et al. "Grid-interface bidirectional converter for residential DC distribution systemsPart one: High-density two-stage topology." IEEE Trans. Power Electron 28.4 (2013): 1655-1666.

4. Khor Sandi, Amir, Mujtaba Ashour, and Hossein Mukhtar. "A decentralized control method for a lowvoltage DC microgrid." IEEE Transactions on Energy Conversion 29.4 (2014): 793-801. 